\title{
RITOURNELLES ET TERRITOIRES PROFESSIONNELS LE CAS DES CONSEILLERS EMPLOI
}

Jean-François Orianne

Éditions de la Maison des sciences de l'homme | «Langage et société »

2015/2 nº 152 | pages 99 à 120

ISSN 0181-4095

ISBN 9782735117536

Article disponible en ligne à l'adresse :

https://www.cairn.info/revue-langage-et-societe-2015-2-page-99.htm

Distribution électronique Cairn.info pour Éditions de la Maison des sciences de l'homme.

(C) Éditions de la Maison des sciences de l'homme. Tous droits réservés pour tous pays.

La reproduction ou représentation de cet article, notamment par photocopie, n'est autorisée que dans les limites des conditions générales d'utilisation du site ou, le cas échéant, des conditions générales de la licence souscrite par votre établissement. Toute autre reproduction ou représentation, en tout ou partie, sous quelque forme et de quelque manière que ce soit, est interdite sauf accord préalable et écrit de l'éditeur, en dehors des cas prévus par la législation en vigueur en France. Il est précisé que son stockage dans une base de données est également interdit. 


\title{
Ritournelles et territoires professionnels Le cas des conseillers emploi
}

\author{
Jean-François Orianne \\ Docteur en sociologie, professeur à l'Université de Liège (ISHS) \\ jforiannedulg.ac.be
}

\section{Introduction ${ }^{1}$}

L'objectif de ce texte est d'utiliser le concept de ritournelle, emprunté à G. Deleuze et F. Guattari, pour analyser la formation d'un groupe professionnel dans le champ des politiques d'emploi en Belgique: les conseillers emploi du FOREM.

Le métier de conseiller emploi naît officiellement, en 2004, par un décret de la Région wallonne. Le Gouvernement wallon accorde la licence exclusive à une nouvelle catégorie de travailleurs du service public d'emploi (FOREM) d'exercer une activité de conseil auprès des demandeurs d'emploi bénéficiaires du Dispositif intégré d'insertion socioprofessionnelle (DIISP). La mission de conseil ou d'accompagnement vers l'emploi remplace en quelque sorte l'ancienne fonction de placement des chômeurs dans l'emploi. Dans un contexte croissant de pénurie de places sur le marché du travail, les placeurs publics sont devenus des conseillers emploi.

Cet article ne propose ni une description ni une analyse du travail des conseillers emploi. Je souhaiterais ici me centrer sur quelques actes de

1. L'écriture de ce texte a grandement bénéficié des discussions que j'ai eues avec Sophie Thunus, Florent Champy, Charles Gadéa et Olgierd Kuty. Je tiens également à remercier les deux lecteurs anonymes de la revue pour leurs commentaires pertinents et constructifs. 
langage significatifs, les ritournelles professionnelles, ces énoncés répétitifs et différenciés par lesquels les conseillers constituent, tracent les bornes de leur territoire professionnel, délimitent un " coin de trottoir ", leur « juridiction» au sens d'Abbott (1988).

Comme le note très justement H.C. Withe, le langage $a$, jusqu'il $y$ a peu et à quelques exceptions près, été largement absent des sciences sociales (White, 2011 : 38). La sociologie des professions ne s'est pas construite autrement. Malgré une littérature foisonnante, rien qu'en France, sur la formation et la dynamique de groupes professionnels (Dubar et al., 2011; Demazière, Gadéa, 2009), peu d'études ont été consacrées spécifiquement au langage des professions. Or, la communication traverse de part en part le travail professionnel. Plus encore, elle le constitue. S'il semble aujourd'hui évident que la sociolinguistique aurait beaucoup apporté à l'analyse des groupes professionnels, constatons cependant que ce linguistic turn est déjà amorcé par les travaux les plus novateurs de la discipline: ils portent sur les stratégies discursives ou rhétoriques professionnelles (Abbott, 1988 : 98-100; Paradeise, 1985), les délibérations intra professionnelles (Champy, 2011), les controverses au sein du discours « savant» (Larson, 1988). Le concept de ritournelle compléterait utilement le tableau, la boite à outils permettant de rendre compte du processus par lequel se forme un groupe professionnel. À partir de ce concept, je tenterai de décrire la formation des conseillers emploi, en tant que groupe professionnel, et d'ouvrir quelques pistes de réflexion et d'analyse plus générale pour la sociologie des professions.

Cette réflexion s'enracine dans une recherche empirique réalisée dans le cadre de ma thèse de doctorat en sociologie auprès de différents groupes professionnels œuvrant dans le champ des politiques d'emploi (Orianne, 2005) $)^{2}$.

2. Le travail de terrain s'est déroulé en Région wallonne (Belgique) entre mars 2001 et juin 200. Nous y avons suivi, dans la quotidienneté de leur travail, une cinquantaine d'agents issus de divers organismes d'insertion. Nous nous sommes principalement centrés sur trois groupes professionnels: les conseillers emploi du FOREM, les conseillers en formation des Carrefours Formation et les travailleurs sociaux des Entreprises de Formation par le Travail. Nous avons réalisé une cinquantaine d'entretiens d'agents $(n=49)$ et une trentaine d'entretiens d'usagers $(n=32)$. Nous avons, en outre, observé de nombreuses interactions entre agents et usagers, dont une trentaine a pu être intégralement enregistrée $(\mathrm{n}=30)$. Nous avons également observé de nombreuses interactions entre agents, dans la quotidienneté de leur travail et dans le cadre particulier de réunions d'équipes, de séances de formation ou de réunions de travail chez des «opérateurs partenaires ». Une seconde phase d'enquête a été réalisée entre avril 2005 et mars 2006 auprès des mêmes acteurs. 
L'article se structure en quatre parties. Je commencerai par présenter les objectifs théoriques: d'une part, tester empiriquement les apports potentiels des théories de la communication réflexive à l'analyse des professions et groupes professionnels; d'autre part, forger un concept sociologique, celui de ritournelle, permettant de saisir finement le processus de formation de groupes professionnels, à partir d'une analyse du discours des acteurs, particulièrement attentive aux « énoncés répétitifs qui tracent des territoires ». Dans un deuxième temps, j'esquisserai brièvement le contexte politique et institutionnel au sein duquel ce travail émerge et s'expérimente. Ensuite, j'analyserai trois ritournelles professionnelles, trois procédés de territorialisation des identités professionnelles et des activités de travail. Je reviendrai, en conclusion, sur la complémentarité du concept de ritournelle avec celui de rhétorique professionnelle.

\section{Objectifs théoriques}

\subsection{Les apports des théories de la communication réflexive à l'analyse des groupes professionnels}

Le groupe professionnel se distingue de l'interaction provisoire sur un lieu de travail, dans la mesure où de simples participants y deviennent des membres. Mais comment se forme le groupe professionnel? Et par quels mécanismes de simples participants deviennent-ils membres d'un groupe?

La sociologie des professions analyse et explique la formation et la dynamique d'un groupe professionnel ou d'une profession à partir de plusieurs variables clés: l'influence exercée par l'État, par les clients, par d'autres groupes concurrents, le développement des connaissances savantes et profanes, la socialisation professionnelle, etc. Si tous ces éléments de réponse impliquent le langage, peu de travaux s'intéressent à la communication en tant que telle. Les théories de la communication réflexive apportent des éléments de réponse intéressants et novateurs dont le sociologue des professions peut se saisir. Pour Luhmann (1999) ou Teubner (1996), la constitution autoréférentielle est une composante clé du processus par lequel de simples participants deviennent membres d'un groupe: selon ces auteurs, la communication réflexive définit les limites du groupe et permet au groupe de se constituer lui-même comme un système d'action autonome. "Alors que les limites de l'interaction se forment pour ainsi dire naturellement par la présence des participants, c'est la communication réflexive qui définit celles du groupe, au sein du système et comme qualité de membre " (Teubner, $1996: 259-261$ ). Le concept de réflexivité est entendu ici comme la capacité d'auto-observation et d'auto-description d'un système d'action. 
Ces travaux issus de la sociologie du droit offrent, me semble-t-il, des pistes intéressantes pour expliquer les propriétés émergentes des groupes professionnels et de l'organisation formelle des professions, sans pour autant tomber dans la " métaphore organiciste " de la personnalité réelle ou d'une conscience collective (comme unité corporelle ou spirituelle): " la réalité sociale de l'acteur collectif consisterait plutôt dans l'autodescription socialement contraignante d'un système d'action organisé, où l'identité s'enchaîne cycliquement à l'action " (op. cit.: 285). Cette approche soutient la thèse selon laquelle le groupe professionnel et l'organisation formelle émergent de l'autonomisation progressive de réseaux d'interaction. Cette orientation théorique, résolument procédurale, renvoie dos-à-dos la thèse " nominaliste » de la fiction ou de la mystification (la profession comme folk concept, chez Becker) et la thèse "substantialiste " de la personnification (l'unité organique de la profession, chez Durkheim). Selon Teubner, l'acteur corporatif n'est ni une fiction, ni un rassemblement d'êtres humains, mais une séquence intense d'événements de communications autoréférentielles et autoproduites: « un collectif provient tout simplement de l'auto-description au sein du système d'action lui-même. Il possédera donc le statut réel (ou fictif) des auto-descriptions sociales » (op. cit.: 262). Le territoire professionnel ne se définit donc pas par le " peuple » des membres, mais bien par le champ de validité de ses normes et par la portée de ses énoncés.

Pour persister, les interactions doivent produire des auto-descriptions garantissant leur caractère réitérable en dépit de la séparation des participants. Une fois que cette auto-description de la qualité de membre en tant que limite du système d'action est effectivement utilisée de façon opérationnelle, le groupe s'est constitué lui-même comme système d'action autonome (Teubner, $1996: 259-261$ ).

Teubner propose de présenter l'autonomie comme un concept gradué et distingue trois niveaux: 1) l'interaction dont les composantes (élément, structure, limite, identité) sont produites socialement par la situation de coprésence; 2) le groupe dont les composantes sont constituées autoréférentiellement; 3) l'organisation formelle dont les composantes sont couplées hypercycliquement ${ }^{3}$.

3. À ce stade supérieur, précise Teubner, l'organisation formelle se rend indépendante de ses membres concrets: désormais, les règles formelles définissent l'appartenance. « Du fait même, l'organisation a gagné un critère maittrisable, lui permettant de contrôler la qualité de membre à elle seule et à l'exclusion de l'environnement » (1996 : p. 262). 


\subsection{Le concept de ritournelle}

Pour décrire le passage de l'interaction (niveau 1) au groupe (niveau 2), pour comprendre comment des travailleurs en interaction forment progressivement un groupe professionnel, le concept de ritournelle semble particulièrement fécond. Désignant "un ensemble de matières d'expression qui tracent un territoire » (Deleuze et Guattari, 1980 : 397), ce concept est utile pour rendre compte du processus de territorialisation d'une activité de travail et d'une identité professionnelle, pour évaluer le degré de constitution et l'étendue d'un territoire professionnel.

Si la ritournelle territoriale passe si souvent dans les ritournelles professionnelles, c'est que les professions supposent que des activités fonctionnelles diverses s'exercent dans un même milieu, mais aussi que la même activité n'a pas d'autres agents dans le même territoire (...). Chez l'animal comme chez l'homme, ce sont les règles de distance critique pour l'exercice de la concurrence: mon coin de trottoir. Bref, il y a une territorialisation des fonctions qui est la condition de leur surgissement comme " travaux » ou " métiers » (Deleuze et Guattari, 1980 : 394).

Les ritournelles délimitent des territoires existentiels, comme le souligne F. Guattari (1992 : 30-32). Les ritournelles constituent des « marqueurs frontières " (au sens de Goffman, 1973 : 55), des " énoncés limites » (au sens de Maingueneau, $2014:$ 151) " placés sur une limite et traitant de la limite ${ }^{4}$.

Chaque individu, chaque groupe, chaque nation s' «équipe " (...) d'une gamme de base de ritournelles conjuratoires. Les métiers et les corporations, de la Grèce antique, par exemple, possédaient en propre une sorte de sceau sonore, une courte formule mélodique appelée " nome ». Ils s'en servaient pour affirmer leur identité sociale, leur territoire et leur cohésion interne; chaque membre du groupe "appartenant " au même shifter sonore, la ritournelle prenait ainsi fonction de sujet collectif et a-signifiant de l'énonciation (Guattari, 1979 : 109).

Mais la ritournelle est aussi directionnelle: elle projette vers de nouveaux agencements territoriaux ${ }^{5}$.

4. "Ces discours ultimes, que nous disons « constituants", se caractérisent au premier chef par la singularité de leur position dans l'univers du discours: ils se situent sur une frontière, celle qui leur permet de parler au nom d'un Absolu qui, lui, ne peut parler qu'à travers eux. (...) Les discours constituants associent en effet étroitement un travail de fondation dans et par leur énonciation, la détermination d'un lieu associé à un corps d'énonciateurs légitimes, et une élaboration de la mémoire " (Maingueneau, 2014 : 151).

5. Quand elle ne cherche plus "la détermination momentanée d'un centre " (idem: 382) mais bien "des composantes pour l'organisation d'un espace », la ritournelle devient « dimensionnelle, elle va vers l'agencement territorial, s’y installe ou en sort. » (idem: 397). 
La profession, le métier, la spécialité impliquent des activités territorialisées, mais elles peuvent aussi bien décoller du territoire pour construire autour d'elles, et entre professions, un nouvel agencement. Une composante territoriale ou territorialisée peut se mettre à bourgeonner, à produire: c'est tellement le cas qu'il faudrait peut-être appeler ritournelle tout ce qui est dans ce cas (Deleuze et Guattari, 1980 : 400-401).

Afin d'importer utilement ce concept philosophique dans l'outillage théorique de la sociologie des professions, il convient avant tout de l'opérationnaliser. En effet, comment s'y prendre pour saisir, capter les ritournelles en situation d'observation ou d'entretien? Comment les repérer dans un matériau empirique (transcriptions d'entretiens, comptes rendus d'observations, enregistrements, notes diverses, etc.)? Deux indices peuvent guider l'analyse des ritournelles: a) leur caractère répétitif et différencié; b) leur fonction territoriale. Dans notre étude, les ritournelles s'apparentent à des refrains ou des rengaines. Elles se jouent, pourraiton dire, sur plusieurs tons, peuvent être légèrement modulées, prendre différentes formes, toujours similaires. Il ne s'agit donc pas exactement d'un énoncé isolé reproduit à l'identique par plusieurs acteurs à plusieurs reprises («nul n’entre ici s'il n'est géomètre »). Il ne s'agit pas non plus d'un thème du discours des acteurs, catégorisé par le sociologue ou par les acteurs eux-mêmes. Chaque ritournelle est une gamme d'expressions: un ensemble d'énoncés similaires, du point de vue de la forme et du contenu, et répétés fréquemment par un collectif d'acteurs. Sa fonction territoriale donne à penser que la ritournelle s'observe d'autant mieux en situation d'incertitude ou de menace, lorsque le territoire est en danger, lorsque les limites sont remises en question. Selon Deleuze et Guattari, la ritournelle apaise, elle est « l'esquisse d'un centre stable et calme, stabilisant et calmant, au sein du chaos » (idem: 382). Comme le note H.C. White, «les identités surgissent d'efforts de contrôle dans un contexte de turbulences (...). La normalité que l'on perçoit est un verni masquant la turbulence des efforts de contrôle par les identités à la recherche d'appuis " (2011: 43-44) $)^{6}$. Selon White, les identités se constituent par le langage, par la communication ${ }^{7}$ : elles recherchent des descriptions des situations (pour elles-mêmes aussi bien que pour les autres) et s'emboîtent, s'encastrent

6. Le contrôle est entendu ici comme ce qui fait émerger de l'ordre; il résulte d'efforts, de tentatives de maitriser un environnement chaotique, de recherche de points d'appui, de points d'ancrage de la part d'identités. «Les identités émergent des turbulences et recherchent le contrôle au sein de bases sociales qui peuvent atténuer l'incertitude" (idem: 62).

7. Sa position théorique est très proche de celle de Luhmann (White, $2011: 236$ ). 
dans des récits (idem: 85$)^{8}$. Les ritournelles constituent des proto-récits, des embryons de récits ou de rhétoriques ${ }^{9}$ professionnelles, qui permettent aux identités professionnelles de se territorialiser.

\section{Mise en contexte}

Le conseiller emploi du FOREM, appartenant au service "Conseil » du Service Public d'Emploi en Région wallonne, exerce un métier qui émerge de lourdes transformations de l'intervention de l'État dans l'organisation du marché du travail: d'une part, le développement de politiques actives d'emploi - en particulier la mise en ouvre de la politique européenne du Parcours d'insertion - et d'autre part, par la ratification de la convention 181 de l'OIT (Organisation internationale du travail) qui autorise les opérateurs privés à offrir des services en matière de placement des travailleurs. La redéfinition à l'échelle européenne de l'objectif des politiques d'emploi en termes d'employabilité, ainsi qu'une nouvelle situation de concurrence entre les intermédiaires publics et privés du marché du travail, sont deux éléments contextuels qui ont directement contribué à mettre un terme au "règne » du placeur public, - une fonction qui vit le jour en 1935 lors de la création de l'Office National du Placement et du Chômage. Le conseiller emploi est un descendant direct du placeur, mais il ne place plus. Il apparait comme la concrétisation la plus aboutie, à ce jour, d'une nouvelle logique d'accompagnement des demandeurs d'emploi, qui s'est progressivement substituée à l'ancienne fonction de placement des chômeurs.

La mise en place du Plan d'Accompagnement des Chômeurs (PAC), en 1993, constitue une première étape clé dans la transformation des pratiques professionnelles par l'introduction d'une logique d'accompagnement ${ }^{10}$. En Région wallonne, au sein de l'office régional wallon de l'emploi et de la formation (FOREM), les Bureaux de Placement se divisent en deux services distincts, à partir de 1995: d'un côté le service "Gestion des offres » et de l'autre le service " Gestion des demandeurs d'emploi ». À la suite de ce premier « choc » organisationnel, la direction

8. Les récits marquent des liens entre des identités, selon White; ils servent à décrire ces liens dans des réseaux; ils structurent les communications.

9. Selon White, les rhétoriques sont des récits qui deviennent des auto-descriptions (ou descriptions partagées) (p. 230); ils forment une théorie populaire en action (p. 235) qui rend les institutions explicites (p. 239).

10. Accord de coopération du 22/09/1992 entre l'État, les Communautés et les Régions concernant le plan d'accompagnement (M.B. du 21/11/1992); A.R. du 02/06/1993 portant financement du plan d'accompagnement (M.B. du 19/06/1993). 
générale du FOREM fait appel à un consultant privé, en 1997, pour

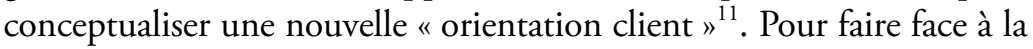
concurrence des acteurs privés du placement et mettre en œuvre les politiques européennes d'employabilité (pilier 1. de la SEE), le consultant (Arthur Andersen) encourage l'Office à se repositionner sur des " activités nouvelles " (comme le coaching), à saisir les " opportunités importantes de développement de nouveaux métiers, dans une société globale de services ». Diverses actions stratégiques sont proposées: l'une d'elles concerne la mise en œuvre de " conseillers entreprises " et de " conseillers emplois ». À un groupe relativement homogène de fonctionnaires du service public d'emploi, définis comme techniciens des processus d'appariement sur le marché du travail, se substituent progressivement de nouveaux collectifs de travailleurs contractuels, aux statuts hybrides et souvent précaires, porteurs de compétences nouvellement valorisées au sein de l'administration, liées à la clinique ou au travail social, à la communication, au coaching. Les conseillers emploi du FOREM ${ }^{12}$ forment un nouveau segment professionnel (Strauss, 2002), une nouvelle génération d'intermédiaires publics (Gélot et Nivolle, 2000). Lors de notre entrée sur le terrain, en mars 2002, les conseillers emploi du FOREM représentent un volume de 209 équivalents temps plein, de niveau d'études $\mathrm{BAC}+3$; ce sont majoritairement des " travailleurs sociaux " (titulaire d'un diplôme d'assistant social, d'assistant en psychologie, d'éducateur, ou de formateur).

Le Gouvernement wallon accorde à ce nouveau groupe professionnel une licence (Hughes, 1996) exclusive pour exercer son activité: il le charge spécifiquement de suivre les bénéficiaires tout au long de leur parcours d'insertion contractualisé (ou contrat crédit insertion) ${ }^{13}$. Le conseiller emploi est défini comme le référent unique chargé d'accueillir, d'informer, d'accompagner, de " coacher " de manière " proactive " et individualisée les demandeurs d'emploi ${ }^{14}$. Sur la base de cette licence officielle, une organisation professionnelle se constitue au sein de l'administration centrale du FOREM, et œuvre à la traduction professionnelle de l'autorisation légale d'exercer, à l'élaboration d'un mandat (Hughes, 1996 : 99-100).

11. Arthur Andersen, FOREM: Projet Ulysse, Rapport Final, mai 1997.

12. La dénomination de la fonction au sein du FOREM a quelque peu évolué: « conseiller particulier du Pôle Emploi » (entre 2000 et 2002), « conseiller en accompagnement professionnel " (2002-2008), et depuis « conseiller emploi ».

13. Gouvernement wallon, Décret du 01/04/2004 relatif au dispositif intégré d'insertion socioprofessionnelle, article 5, Namur.

14. Avant-projet de décret relatif au dispositif intégré d'insertion socioprofessionnelle, article 5, Namur, 18/07/2002: commentaires des articles, p. 3-7. 
Il ressort de cette définition organisationnelle du métier deux éléments essentiels, étroitement liés: la fonction de diagnostic et l'image d'un généraliste qui renvoie à des spécialistes ${ }^{15}$.

\section{Une analyse des ritournelles professionnelles}

\subsection{Ritournelle (R1) : « Nous, on n'est pas l'ONEM,} on n'est pas des flics de l'insertion »

Comme le rappelle C. Dubar (2000), l'identité est de part en part relationnelle, elle se construit dans la relation à l'autre: à un autrui singulier (les autres) et à un autrui généralisé (Mead), à l'Autre (Lacan), en clair, à l'institution ${ }^{16}$. Quelle est l'identité des conseillers emploi du FOREM? Comment se définissent-ils? Auprès de qui et contre qui s'identifient-ils? "Nous, on n'est pas l'ONEM », "on n'est pas des flics de l'insertion", ne cessent-ils de répéter.

Cette première ritournelle exprime une différenciation fonctionnelle croissante entre deux catégories d'agents du service public d'emploi, ceux qui appartiennent à l'administration régionale (FOREM) et ceux de l'administration fédérale (ONEM) : d'un côté, le soutien à l'employabilité des personnes sans emploi, de l'autre, le contrôle des bénéficiaires de l'assurance chômage ${ }^{17}$. La ritournelle constitue une voie principale de territorialisation des activités de travail: « le territoire, c'est d'abord la distance critique entre deux êtres d'une même espèce: marquer ses distances. » (Deleuze et Guattari, 1980 : 393). Ce refrain esquisse les contours de l'espace professionnel du conseiller emploi. Comme le confie cette conseillère de Libramont en entretien, « moi, je ne suis pas l'ONEM, je ne suis pas là pour qu'ils (les demandeurs d'emplois) me rendent des comptes ». Cette ritournelle s'exprime en entretien, mais également en réunion d'équipes, et surtout face au public, comme en témoignent ces trois courts relevés d'interactions observées entre un conseiller emploi du FOREM et un chômeur.

15. Le FOREM, L'organisation passe à l'action, Brochure institutionnelle, Département Communication et marketing, novembre 2002, p. 14.

16. Selon Dubar, l'identité " n'est autre que le résultat à la fois stable et provisoire, individuel et collectif, subjectif et objectif, biographique et structurel, des divers processus de socialisation qui, conjointement, construisent les individus et définissent les institutions» (Dubar, 1991 : 113). L’identité est comme un « espace-temps générationnel » selon Dubar, un « territoire existentiel» selon Guattari (1992).

17. Accord de coopération du 01/07/2004 entre l'État Fédéral, les Régions et les Communautés, relatif à l'accompagnement et au suivi actifs des chômeurs; Arrêté royal du 4/07/2004 portant modification de la réglementation du chômage à l'égard des chômeurs complets qui doivent rechercher activement un emploi (M.B. 09/07/2004). 
Moi, je ne suis pas l'ONEM, donc je ne suis pas là pour vérifier si tu fais réellement des recherches ou pas. Je suis là pour t'accompagner et t'aider. (FOREM Libramont)

Moi, je ne suis pas un gendarme, je ne suis pas l'ONEM. Moi, je suis là pour essayer de donner quelque chose, je ne suis pas là pour épier, obliger, surveiller, je n'ai pas le temps et ce n'est pas du tout mon travail. (FOREM Libramont)

Moi, je suis là pour entendre ton projet. Si ton projet c'est: écoutez, moi pour l'instant, la recherche emploi ça ne m'intéresse pas, j'ai envie de me consacrer trois-quatre mois à ne rien faire, moi je peux l'entendre aussi. Je ne suis pas l'ONEM, je ne suis pas là pour te surveiller, si la recherche d'emploi ce n'est pas dans ta tête, on travaillera quand elle sera dans ta tête. (FOREM Libramont)

Cette ritournelle semble remplir deux fonctions essentielles. Face au public, elle permet au conseiller d'informer l'usager sur la mission de conseil au sein du FOREM, de re-spécifier la relation de travail (modalités, objectifs, évaluation, etc.) du conseiller emploi. Mais dans l'entre-soi, lors d'un colloque, d'une formation ou d'une réunion d'équipe, cette ritournelle de distinction avec un groupe professionnel "voisin " (les facilitateurs de l'ONEM) permet de construire une identité collective, de se positionner " avec quelque peu d'humanité " par rapport au contrôle des chômeurs.

Le plan d'accompagnement des chômeurs pour moi, j'ai appris que ça existait, c'est que c'était une façon de trier les chômeurs qui avaient un an de chômage. De trier et de dire: " mais écoutez, ceux qui cherchent pas et qui ne répondent pas aux critères qu'on va établir, on va le dire à l'ONEM ». (...) Ça été ressenti comme ça au niveau du public, comme une procédure d'exclusion. Et à la limite je vais dire, le jour où on s'est présentées pour le boulot, on n'en savait rien. Et puis, quand on a été sélectionnées à 3, donc on était 3 licenciées, il y avait eu X, il y avait $\mathrm{Y}$ et moi, - on avait été crier dans la rue avec la FGTB pour dire que c'était une abomination -. Et puis, on s'est retrouvées dedans. Et alors on s'est dit: "bon écoute, on se trouvait pas mal, on se trouvait très sociales par rapport à notre objectif de départ. Dans notre façon de réagir et de penser ". Et on s'est dit: " écoute, si on laisse la place à d'autres, on ne sait pas dans quelle dérive ça va aller » Et donc, si on peut compter sur notre jugement à toutes les 3, on pourra au moins donner dans ce procédé un... quelque peu d'humanité. (...). Et donc on a commencé à construire ce métier qui n'existait pas avant. (Entretien de A, conseillère emploi, FOREM Libramont)

Cette valorisation de l'aide, du soutien, de l'accompagnement individualisé, par rapport au contrôle, constitue un axe central de l'identité professionnelle du conseiller. L'approche humaniste du traitement des 
chômeurs efface les dernières traces d'une conception coercitive du métier (ancienne filiation avec l'ONEM) et esquisse les contours d'un nouveau territoire professionnel, en vis-à-vis de celui des facilitateurs. Comme le précise ce conseiller du FOREM de Liège, « il y a une certaine humanité qu'on trouve au FOREM qui n'est pas incompatible, je crois, avec le professionnalisme ».

Les ritournelles sont des lignes de différenciation (nous/eux). Comme le souligne très justement $\mathrm{M}$. Glady, « l'analyse de la distribution actantielle, par laquelle le locuteur convoque et organise les rapports entre les sous-groupes professionnels, les différents profils ou même les métiers à l'intérieur du secteur de l'accompagnement (...) rend compte de l'univers imaginaire des professionnalités, sous-tendu par des rapports d'identification et de différenciation entre actants. (...) À la fois réels et imaginaires, ces rapports de place sont opérants dans la construction des interactions quotidiennes " (Glady, 2011 : 33). Ces énoncés sont donc porteurs d'effets pragmatiques en ce qu'ils autorisent et orientent de nouvelles pratiques au sein du service public de l'emploi: en regard des facilitateurs de l'ONEM, les conseillers emploi du FOREM se définissent comme les " généralistes de l'employabilité », dont la tâche principale est d'accompagner des individus qui travaillent leur employabilité (dans le cadre d'entretiens individualisés ou de séances d'information collectives, de groupes de discussion ou d'ateliers d'écriture de lettres de motivation et de C.V., de simulations d'entretiens d'embauche filmées, de mises en situation professionnelle, etc.). Comme nous l'avons montré par ailleurs, ce travail nécessite de prendre en compte différents « troubles de l'employabilité ${ }^{18}$, de les analyser, d'en dresser la symptomatologie, de les diagnostiquer dans une approche globale de l'individu, et de renvoyer les individus à des spécialistes compétents (cf. Orianne, 2005).

Est-ce que ça se dessine clairement, pour toi, la spécificité du CAP dans l'ensemble de cette structure? C'est un peu le médecin généraliste qui va renvoyer à des spécialistes. Tu sais c'est vraiment rattaché au corps médical; je trouve que l'image n'est pas si mauvaise que ça. (...) Donc on va faire tout un travail de visualisation, de relevé de tout... - enfin si on parle le langage médical -, le relevé de toute la symptomatologie qui nous permet d'établir à terme un diagnostic. (Entretien de $\mathrm{B}$, responsable des conseillers emploi, FOREM Libramont)

18. Par exemple: le manque de confiance en soi, la maîtrise insuffisante des techniques de recherche d'emploi, l'absence de projet professionnel, le manque d'expérience professionnelle, divers « troubles » de l'orientation professionnelle ou de la motivation, les «problèmes » familiaux, etc. 
Cette image du " généraliste » ou du " médecin de famille » - induite par l'administration centrale du FOREM (cf. infra) - est fortement relayée par les professionnels de terrain pour définir, expliquer (à un demandeur d'emploi comme à un chercheur) le contenu, le sens de leur travail. L'image du généraliste de l'employabilité sert de balise, de point d'appui pour ces travailleurs du service public, anciens placeurs ou nouveaux entrants, qui expérimentent, en situation d'incertitude, cette nouvelle mission.

\subsection{Ritournelle (R2) : « Le sens de notre travail, ce n'est pas de trouver des solutions »}

Une deuxième ritournelle concerne le sens de leur travail. Qu'est-ce qu'un métier d'accompagnement vers l'emploi? "Le sens de notre travail, - disent fréquemment les conseillers emploi -, ce n'est pas de trouver des solutions ». Par ce refrain, ils (re-)tracent, (re-)spécifient les bornes, les limites de leur intervention. Le conseiller emploi n'est plus un placeur, il n'est pas là pour mettre à l'emploi. L'expression force l'évidence, frise le lieu commun. En effet, leur mission ne consiste pas à résoudre les "problèmes » des demandeurs d'emploi - le principal problème étant qu'ils n'ont pas d'emploi. Les professionnels de l'insertion n'étant pas responsables du chômage, du manque de place(s) sur le marché du travail, c'est à un autre niveau que se situe leur intervention: elle vise non pas à placer l'individu dans un emploi, à lui proposer une solution toute faite, mais à le faire travailler son employabilité, sa capacité à trouver un emploi, dans le cadre d'entretiens individualisés ou de séances d'information collectives, de groupes de discussion ou d'ateliers d'écriture de lettres de motivation et de C.V., de simulations d'entretiens d'embauche filmées, de mises en situation professionnelle, etc.

La ritournelle s'observe en réunion de travail. À titre d'exemple, lors d'une réunion du "pôle particulier " tenue à Libramont le 17 décembre 2002, ce « refrain » concluait, sous la forme d'une boutade, une réflexion menée collectivement sur la nature et la finalité de l'intervention des conseillers particuliers de FOREM Conseil: "si vous avez un problème d'emploi, venez avec des solutions et le FOREM vous aidera ». Sur un document de travail (le vade-mecum de l'accompagnement), on peut lire la mention en gras: "notre expertise ne consiste pas à trouver une solution rapide! ». Le conseil n'est pas une solution toute faite, apprennent ces agents dans le cadre de formations organisées au siège central de l'administration: la solution ne peut émerger que d'un processus long, un «trajet d'employabilité », que mène le demandeur d'emploi. 
Lors d'un entretien, cette conseillère de Libramont reprend: « ne pas devoir trouver une solution à tout le monde".

Mais c'est vrai que je pense que je parle comme ça aujourd'hui parce que je pense que la dernière formation que je suis allée suivre, je disais tantôt le cadre d'identité professionnelle, ça m’a permis de ne plus devoir absolument trouver une solution à tout le monde. (...) J'arrive à moins me culpabiliser. (Entretien de C, conseillère emploi, FOREM Libramont)

Conjurer le sort (des chômeurs) pour moins « culpabiliser ». La ritournelle exprime ici un déplacement normatif et éthique important que génère la mise en œuvre de politiques actives d'emploi : l'État, à travers ses acteurs de première ligne, ne se présente plus comme responsable de l'emploi ou du non-emploi des individus, selon la convention keynésienne de plein emploi (Salais et al., $1986: 239$ ), il investit dans la responsabilisation des individus quant à leur employabilité, et il charge des professionnels d'accompagner ces individus travaillant leur propre employabilité.

Moi je dis: mon truc, c'est d'écouter la personne, de voir un petit peu où elle en est par rapport à son projet professionnel. Et en fonction de ça, si elle le souhaite, moi je peux lui donner un petit coup de pouce pour que... pas nécessairement décrocher un boulot, mais pour y voir plus clair et voir ce qu'il faut faire pour décrocher ce fameux boulot. (...) C'est ça que je disais, au début, je voulais absolument que les gens travaillent. Et puis même si la personne trouvait du boulot pour te faire plaisir, quinze jours, trois semaines après, il était de nouveau sans emploi. Et c'est de nouveau dans cet engrenage parce qu'il n'était pas prêt. Moi je dis ils ont 18 ans, c'est leur choix, mais je les sensibilise au fait que... voilà... que c'est important... (Entretien de D, conseillère emploi, FOREM Libramont)

Si pour certains, le but de leur travail ce n'est pas forcément que les gens travaillent, pour d'autres, de manière plus tranchée, le sens du travail du conseiller, ce n'est pas de trouver des solutions, pas même d'en chercher. Comme l'explique cette conseillère, donner un emploi, une solution toute faite, c'est la facilité, c'est " envoyer le mec au casse-pipe ": si tu donnes un emploi à un chômeur, il travaillera un jour, si tu lui apprends à pêcher des emplois, il travaillera toute sa vie.

Or, on n'a pas une vocation d'orientation, on a une vocation qui est le diagnostic, et quelque part c'est une proposition de piste, une proposition de priorité aussi dans ces différentes pistes, ... Dans le terme orienter comme on l'entend d'habitude, je crois que c'est pas du tout ça. Et qu'au contraire, ce serait même une dérive à ne pas prendre. Tu sais, de se contenter pour finir de dire: « ben, qu'est-ce que vous voulez? Ah bon, voilà. On va vous orienter vers tel service ». Et pourquoi est-ce que je dis ça, parce que justement la dérive, la grosse dérive à éviter dans notre métier, c'est même pas de trouver une solution, c'est même de la chercher. On est pas là pour parler 
en termes de solutions. Pour un tas de raisons. Vraiment pas. Parce que de nouveau, c'est la facilité, la solution. C'est la petite semelle, quoi. C'est: tu viens chez moi, tu as un problème, je te donne ta solution, tu pars heureux. (...)

C'est facile (...) de sortir une offre d'emploi de sous la table et de dire " présente-toi là ». Et même si ça marche, ce n'est pas notre métier à nous. C'est pas pour ça qu'on est fait en tout cas. J'ai pas dit qu'on le ferait pas. Mais je trouve ça très insignifiant par rapport à ce que tu peux amener. (...) Tu n'as pas testé ses moyens de mobilité etc. pour accéder à un emploi, tu ne sais absolument pas vers quoi il veut aller, et donc c'est très bien s'il se présente à son premier job, mais il y a d'autres chances que ça marche (?) comme ça. Ou alors il va dire oui à l'emploi, il n'est pas capable d'y aller demain parce qu'il n'a pas la bagnole. T'as rien testé, quoi. T'envoies le mec au casse-pipe. Pour moi, c'est ça. Et c'est la grosse différence entre ce que les gens viennent chercher et ce qu'on leur propose, je crois. (Entretien de A, conseillère emploi, FOREM Libramont)

En définissant le métier par ce qu'il n'est pas, l'acteur livre ici, dans la dernière phrase, de manière presque anodine, une des dimensions essentielles de son métier: l'intense travail de traduction que le conseiller opère à partir des attentes des individus (ce que les gens viennent chercher) pour les reformuler en projets, ou du moins en services (ce qu'on leur propose). Si, en théorie, l'approche du conseiller doit se centrer sur les besoins de l'individu (cf. infra, licence et mandat), en pratique, les choses ne sont évidemment pas si simples: l'individu a-t-il conscience de ses besoins réels? Ne confond-il pas besoins et envies ou rêves? Exprime-t-il des besoins auxquels le FOREM peut répondre?

À Seraing, souvent on a des questions cachées. Ils viennent pour un CV et puis on se rend compte qu'il y a des tas d'autres choses, ça pose des tas de problèmes de logement, de famille, ils sont complètement désorientés. Le $\mathrm{CV}$ est parfois le prétexte à plein de trucs... Maintenant, moi je dis que $100 \%$ de satisfaction c'est impossible, parce qu'il y a des tas de gens qui viennent: je voudrais un emploi, mais franchement je ne saurais même pas vous dire ce qu'il y a comme offre d'emploi au FOREM et c'est vrai, je ne le sais pas. Je ne veux pas le savoir non plus parce que c'est pas du tout mon objectif. Je ne cherche pas un travail pour les gens. J'essaie de rendre les gens autonomes ou donner au moins toutes les cartes aux gens pour qu'ils aient les moyens de trouver l'emploi qui leur convient et pas l'emploi que je pourrais leur dénicher, un truc comme ça. (Entretien de E, conseiller emploi, FOREM Liège)

Comme en témoigne la fin de cet extrait d'entretien, cette ritournelle est encastrée dans un discours, celui de l'autonomie, qui semble traverser plus globalement le secteur de l'insertion socioprofessionnelle. Ce discours sur l'autonomie - dont plusieurs auteurs ont souligné la prégnance, 
par exemple dans le champ de la santé mentale, de l'école, des politiques sociales ou encore du travail - constitue pour les conseillers emploi un appui important pour construire leur métier. Le travail sur l'employabilité du demandeur d'emploi consiste à motiver, intéresser la personne sans emploi à se mettre au travail sur elle-même. La fabrication d'un être autonome (en recherche d'emploi) apparaît comme l'objectif principal $\mathrm{du}$ travail d'insertion, et pas uniquement pour les conseillers emploi du FOREM, comme en témoigne l'exemple ci-dessous:

FOREM :

C'est l'autonomie dans la recherche d'emploi qui est visée (Liège).

L'autonomie de la personne en recherche d'emploi : c'est une finalité (...) Tu sais... c'est redonner confiance, leur proposer de devenir acteurs... Et certains le sont déjà... (Arlon)

C'est essayer de les rendre autonomes tout simplement. De leur dire: mais voilà, maintenant tu as toutes les cartes en main... voilà, à toi de jouer (Libramont).

Carrefour Formation:

Je crois au fond ici... je sais que nous ici aussi on a un objectif d'autonomie, on aimerait que les gens soient autonomes (Liège).

C'est bien de vouloir des personnes autonomes, mais il faut leur donner les moyens d'être autonomes. On ne les rend pas autonomes comme ça en claquant des doigts (Liège).

Entreprise de Formation par le Travail:

L’objectif général poursuivi réside bien entendu dans l'acquisition d'une autonomie personnelle, d'une socialisation au monde du travail (Le Trusquin EFT, rapport d'activité 1998 : p. 6).

L'objectif, c'est de permettre à des jeunes d'être autonomes dans leur recherche d'emploi (Marloie).

L'origine de ce discours de l'autonomie, au sein du champ de l'insertion, et en particulier des conseillers emploi du FOREM, peut être éclairée par au moins deux éléments étroitement liés. On notera, d'une part, la montée en puissance, depuis une vingtaine d'années, d'un nouveau référentiel sectoriel (Muller, 2000) dans le champ des politiques d'emploi, porté par les institutions publiques (européennes, fédérales, régionales) et structuré autour de notions hautement mobilisatrices, telles l'activation, l'employabilité, le life long learning, l'empowerment, ou encore l'adaptabilité: le champ lexical de la stratégie européenne pour l'emploi sous-tend (et permet de véhiculer) une conception de l'individu comme " acteur " de son parcours, responsable de ses choix, de ses projets, "entrepreneur de lui-même ", auquel l'action publique doit apporter des ressources "sur mesure ", adaptées à ses besoins (Vandenbroucke, 1999). On rappellera, 
d'autre part, les transformations organisationnelles à l'œuvre au sein du FOREM (cf. infra: point 2), dans un contexte " européanisé » de gestion mixte du marché de l'emploi: délaissant progressivement le placement (à la concurrence des agences privées), le service public de l'emploi se repositionne sur l'accompagnement des chômeurs et, pour ce faire, recrute, par vagues successives ${ }^{19}$, des travailleurs sociaux, et les forme en interne aux techniques de l'accompagnement individualisé (mise en projet, entretien de diagnostic, assertivité, coaching, etc.).

\subsection{Ritournelle (R3) : « Nous, on ne fait pas du quantitatif »}

Que font les conseillers emploi? "Nous, on ne fait pas du quantitatif ", répètent-ils constamment. Cette troisième ritournelle constitue, pour les conseillers emploi, un rempart qui les protège du regard extérieur, de l'évaluation externe de leur travail. Ce refrain exprime une quête d'autonomie professionnelle du groupe par rapport à l'État qui les mandate.

Si tous les métiers luttent en principe pour obtenir un monopole sur l'exercice du travail qui est le leur, le droit d'exercer son autonomie, en toute légitimité, n'est reconnu qu'à la profession, précise Freidson (1984). Qu'en est-il des intermédiaires publics du marché du travail, en particulier des conseillers emploi du FOREM (Orianne et Maroy, 2008) ? Leur travail ne semble pas reposer sur un ensemble de qualifications et de connaissances hors du commun. En outre, les autorités publiques exercent un contrôle important sur l'organisation sociale et économique de leurs activités. Cependant, elles leur laissent simultanément le soin de définir le contenu de leur travail, ce qui, selon Freidson, est l'élément décisif de l'autonomie professionnelle, d'une autonomie de réflexion (Champy, 2011).

On a beaucoup travaillé sur la quantité pendant des années, et dans mes discours, j'arrête pas de recentrer tout sur la qualité. (...) Tu vois ce que je veux dire? (...) Et donc à tout moment il faut rappeler: " attention, personne n'est capable de prendre 8 personnes par jour dans un premier entretien individuel et de le faire correctement. " Là ce sont des critères de qualité qu'on a fixés, à savoir de faire un repérage complet sur tous les axes de la personne etc. Personne n'est capable de faire ça correctement avec 10 individus par jour. Ça non, c'est clair. Et c'est moi qui me charge de dire combien de personnes on prend... C'est quasi une mentalité, une philosophie, je sais pas comment dire... (Entretien de B, responsable du service des conseillers emploi, FOREM Libramont).

19. Principalement en 1993 et en 2004, lors de la mise en œuvre des deux plans d'accompagnement des chômeurs. 
Les conseillers emploi définissent et protègent leur activité, en situation d'incertitude, par une série de formules incantatoires. Celles-ci les protègent de la hiérarchie de l'administration, du ministre de tutelle, qui leur demandent régulièrement des comptes, des rapports statistiques de leur activité. Face à cette pression à l'accountability, les conseillers emplois se sentent menacés - à l'instar de leurs "clients "-, en permanence sous la loupe d'évaluateurs externes, d'experts, de décideurs politiques, de chercheurs qui évaluent, contrôlent, compilent, commentent les résultats (chiffrés) de leur travail.

C'est plus au niveau quantitatif... C'est un peu dommage parce que je pense qu'il y a vraiment une qualité de service offerte chez nous qui ne transparaît pas toujours dans le quantitatif, dans les statistiques. (...) Je dirais pour le qualitatif, la préoccupation existe bel et bien, tu peux noter ça. Mais... il y a le qualitatif au niveau individuel, c'est le travail qu'on fait chaque fois qu'on est face à une personne. Et puis, il y a le qualitatif qui passe dans la quantité... quand il faut rendre des comptes par rapport à l'institution (Entretien de F, conseillère emploi, FOREM Liège).

R. Salais a souligné la dégénérescence instrumentale d'une politique des indicateurs, menée actuellement en Europe, en particulier de la méthode ouverte de coordination. "Il existe empiriquement et politiquement suffisamment d'obstacles à un emploi effectif et efficace de la méthode au sein de la SEE pour que l'ambition de l'étalonnage se réduise in fine à une simple méthode de scores (...). L'objet du jeu (au sens de la théorie des jeux) est le rendement statistique des politiques d'emploi nationales, appréciées du point de vue des indicateurs " (Salais, 2004 : 288). Par cette ritournelle, les conseillers emploi se démarquent, sur un plan identitaire, de cette " politique des indicateurs » instrumentale et autoréférentielle, d'un traitement statistique du chômage (Orianne et Conter, 2007). La qualité, c'est résister!

Cette troisième ritournelle est à la fois un acte de résistance aux orientations dominantes du champ et une revendication d'autonomie professionnelle. L'énonciation marque ici des " processus défensifs de mise à distance du réel pour s'en protéger ", des "processus de mise à l'écart des menaces de transformation de l'exercice du métier» (Glady, 2011 : 35). Comme le souligne M. Glady, un danger guette la profession: « celui d'une perte de la qualité de prise en charge du chômeur sous l'effet de contraintes de volume de traitement; et par conséquent, celui d'une perte d'autonomie des consultants dans l'organisation de leur travail et la répartition des tâches à l'intérieur de l'équipe " (p. 39).

Ah ben, il faut prendre le temps! Maintenant c'est vrai que dernièrement on nous a dit que c'était... qu'on privilégiait la qualité à la quantité. Donc 
maintenant c'est vrai qu'il y a des gens qu'on ne verra jamais... Non. Pour ça, maintenant, nous, on ne se tracasse plus, c'est qualité et pas quantité (Entretien de G, conseillère emploi, FOREM Arlon).

Par rapport au travail, je crois que le message est clair: l'accent est mis sur la qualité et pas sur la quantité, même si effectivement on doit rentrer un grand nombre de dossiers, je sais bien qu'on n'y fera pas face du jour au lendemain, mais voilà (Entretien de H, conseiller emploi, FOREM Arlon).

Oui, mais on ne saurait pas voir tout le monde parce qu'on n'est pas assez de personnel. C'est impossible. Même avec la meilleure volonté du monde... et moi je préfère la qualité que la quantité. On a toujours décidé « qualité » de toute façon. Et maintenant on vient de nous dire qu'on pouvait poursuivre. X (le coach) est allée en réunion à Charleroi. Et Charleroi lui a donné l'accord (Entretien de D, conseillère emploi, FOREM Libramont).

Ce dernier extrait renvoie à l'argument souvent mobilisé par les agents pour justifier leur position: de toute façon, ils n'ont pas les moyens de faire du quantitatif, de faire du chiffre, de voir tout le monde... Comme le rappelle fréquemment, avec humour, ce conseiller de Virton à ses clients: "je ne suis pas payé au rendement, donc que vous ayez ou non un boulot, ça ne change strictement rien pour moi ". On trouve ici une synthèse des trois principales ritournelles du conseiller: il ne met pas à l'emploi, il n'est pas un " flic » de l'insertion, il ne fait pas du chiffre.

Les ritournelles du conseiller agissent à la fois comme des balises et des barricades. Elles servent de repères, d'orientations de sens pour l'action (Ramognino, 2007) et protègent le groupe des invasions profanes (l'orientation client), de la pression concurrentielle d'autres groupes professionnels (comme les facilitateurs de l'ONEM ou encore les placeurs privés qui, eux, "font du chiffre »), de l'intrusion des pouvoirs publics (l'évaluation externe). La forme négative de ces trois ritournelles est intéressante car elle indique le caractère polyphonique ou dialogique de ces énoncés. «On sait (...) que le propre de la négation est que l'on puisse déchiffrer en elle l'assertion de ce qu'elle nie et qu'il s'agit d'une forme de dialogisme montré » (Glady, 2011 : 39). Chaque ritournelle est une réfutation par négation. "Appelée aussi négation polémique, (elle) est une négation qui réfute une affirmation qui est faite - ou qui serait faite, c'est là un point essentiel - par autrui » (Krieg-Planque, 2012 : 211). Elle constitue donc une forme particulière d'interdiscours (au sens de Pêcheux), de polyphonie (au sens de Ducrot), de dialogisme (au sens de Bakhtine) ${ }^{20}$, dans la mesure

20. Pour une présentation synthétique de ces trois notions voisines : lire Krieg-Planque (2012 : 186-213) ou Leimdorfer (2010: 173-186). 
où la ritournelle réfute un discours, le discours de l'autre (celui de l'usager, des facilitateurs, des placeurs privés, des pouvoirs publics, des médias, des chercheurs, etc.). En effet, la ritournelle ne nie pas un contenu; elle convoque à l'intérieur du discours un autre discours et façonne (en discours) une conflictualité, trace une ligne de démarcation.

\section{Conclusion : ritournelles et rhétoriques professionnelles}

L'objectif de ce texte était de tester empiriquement le concept de ritournelle, en suivant les apports des théories de la communication réflexive (en particulier, le modèle de Teubner). Ce concept s'est révélé fort utile, en complément d'une théorie générale des professions, pour étudier la formation du groupe des conseillers emploi en Région wallonne.

Lactivité discursive et délibérative des professionnels constitue l'objet principal de l'étude: prendre au sérieux le discours des professionnels, compte tenu de sa fonction territoriale. Le langage ne constitue pas seulement un médium universel pour coordonner les actions (Mead, 2006), il est aussi une procédure de territorialisation des identités professionnelles, comme l'a bien montré Goffman. Les ritournelles sont des actes de langage "réflexifs " qui à la fois décrivent et constituent in situ un ordre social: celui du traitement clinique des chômeurs qui repose sur le diagnostic des " troubles de l'employabilité ».

Trois ritournelles ont été analysées pour étudier la construction d'une identité professionnelle, la stabilisation d'un système d'action, allant de l'interaction provisoire entre participants à la constitution d'un groupe.

On peut, à ce stade, préciser la distinction conceptuelle entre la ritournelle et la rhétorique professionnelle. La ritournelle est principalement orientée vers le groupe: elle vaut surtout pour soi. Les ritournelles sont auto-objectives dans la mesure où le groupe reconnaît l'« efficacité magique " (Bourdieu, $2001:$ 107) que le mot d'ordre ou le cri de ralliement prétend exercer. Et c'est précisément en vertu de cette caractéristique qu'il convient d'interroger la portée territoriale de la ritournelle, dont la nature auto-objective (performative) territorialise les interactions. La rhétorique, à l'inverse, est orientée vers autrui (Paradeise, 1985; Abbott, 1988): il ne s'agit plus de se rassurer, de se persuader, mais bien de convaincre.

En situation d'incertitude, le discours n'est pas encore un mécanisme de revendication mais plutôt un signe de ralliement ou de repli, la production discursive n'est pas dirigée vers autrui mais vers la recherche d'une position d'équilibre au sein d'un contexte menaçant. Contrairement à la rhétorique professionnelle, dont la fonction est persuasive, stratégique, la ritournelle professionnelle a une fonction 
identitaire (auto-persuasive) et territoriale (auto-constitutive). Comme le note très justement $\mathrm{D}$. Segrestin, «l'identité est une fin en soi telle que le groupe professionnel peut freiner la satisfaction de ses intérêts et ne pas poursuivre l'objectif du 'toujours plus' (c'est-à-dire ses intérêts), pour viser au contraire le 'toujours là' " (Segrestin, 1985 : 91, 210; cité dans Kuty, $1991: 16$ ).

Une analyse sociologique du langage des professions devrait tenir compte de ces deux dimensions essentielles du discours professionnel: l'une relève de la ritournelle, elle est identitaire et orientée vers soi; l'autre relève de la rhétorique, elle est stratégique et orientée vers autrui. Dans une conception élargie du concept d'identité professionnelle, telle qu'on la trouve chez C. Dubar (1991) à travers notamment la notion de "stratégie identitaire ", on pourrait soutenir l'idée selon laquelle la ritournelle œuvre à la construction d'une identité collective pour soi, alors que la rhétorique constitue un vecteur de stabilisation et de promotion de cette identité collective pour autrui. En reprenant le concept gradué d'autonomie développé par Teubner, on peut émettre l'hypothèse selon laquelle la ritournelle exprimerait le passage de l'interaction au groupe professionnel, alors que la rhétorique exprimerait davantage, au sein d'un système d'action relativement autonomisé, le passage du groupe à l'organisation formelle d'une profession.

\section{Références bibliographiques}

Abbott A. (1988), The System of Professions. An Essay on the Division of Expert Labor, The University of Chicago Press, Chicago-London.

Bourdieu P. (2001), Langage et pouvoir symbolique, Seuil, Paris.

Champy F. (2011), Nouvelle théorie sociologique des professions, PUF, Paris.

Deleuze G. et Guattari F. (1980), "De la ritournelle », dans Mille plateaux, Capitalisme et schizophrénie 2, Les Éditions de Minuit, Paris: p. 381-433.

Demazière D. et Gadea C. (2009), Sociologie des groupes professionnels. Acquis récents et nouveaux défis, La Découverte, Paris.

Dubar C. (1991), La socialisation: construction des identités sociales et professionnelles, Armand Colin, Paris.

Dubar C., Tripier P., Boussard V. (2011), Sociologie des professions, Armand Colin, Paris. 
Freidson E. (1984), La profession médicale, Payot, Paris.

Gélot D. et Nivolle P. (dirs) (2000), Les intermédiaires des politiques publiques de l'emploi, Cahier Travail et Emploi, La documentation Française, Paris.

Glady M. (2011), «Pratiques d'accompagnement des demandeurs d'emploi. L'apport de la sociologie du langage ", Langage et Société, n ${ }^{\circ} 137$, p. $17-45$.

Goffman E. (1973), "Les territoires du moi ", in La mise en scìne de la vie quotidienne. 2. Les relations en public, Les éditions de Minuit, Paris: p. 43-72.

Guattari F., (1979), Linconscient machinique. Essais de schizo-analyse, Encres, Paris.

- (1992), Chaosmose, Galilée, Paris.

Hughes E.C. (1996), Le regard sociologique. Essais choisis, Textes rassemblés et présentés par Jean-Michel Chapoulie, Éditions de l'École des Hautes Études en Sciences Sociales, Paris.

Krieg-Planque A. (2012), Analyser les discours institutionnels, Armand Colin, Paris.

Kuty O., (1991), «La problématique de la création des valeurs dans la sociologie contemporaine des professions. Le cas des professionnels de première ligne ", Sciences sociales et santé, vol. 9, n² 2, p. 5-30.

Larson M. S. (1988), «À propos des professionnels et des experts ou comme il est peu utile d'essayer de tout dire ", Sociologie et sociétés, vol. XX, $\mathrm{n}^{\circ} 2$, p. $23-40$.

Lascoumes P., Le Bourhis J.-P., (1998), « Le bien commun comme construit territorial. Identités d'action et procédure. ", Politix, vol. 11, n 42, p. 37-66

Leimdorfer F. (2010), Les sociologues et le langage, Éditions de la Maison des Sciences de l'Homme, Paris.

Luhmann N. (1999), Politique et complexité, Cerf, Paris.

Maingueneau D. (2014), Discours et analyse du discours, Armand Colin, Paris.

Mead G. H. (2006), L'esprit, le soi et la société, PUF, Paris (édition originale, 1937). 
Muller P. (2000), «L'analyse cognitive des politiques publiques: vers une sociologie politique de l'action publique ", Revue française de science politique, vol. $50, \mathrm{n}^{\circ} 2$, p. 189-208.

Orianne J.-F. (2005), Le traitement clinique du chômage, Thèse de doctorat, UCL, Louvain-la-Neuve.

Orianne J.-F., Conter B. (2007). « Les politiques d'employabilité en Belgique: traitement clinique des chômeurs et traitement statistique du chômage ", Recherches Sociologiques et Anthropologiques, XXXVIII (2), 175-190.

Orianne, J.-F., Maroy, C. (2008). «Esquisse d'une profession consultante. Les intermédiaires du marché du travail en Wallonie », Formation Emploi, 102, 21-40.

Paradeise C. (1985), «Rhétorique professionnelle et expertise ", Sociologie du travail, $\mathrm{n}^{\circ} 1-85$, p. 17-31.

Ramognino N. (2007), « Normes sociales, normativités individuelle et collective, normativité de l'action ", Langage et Société, n 119, p. 13-41.

Salais R. (2004) «La politique des indicateurs. Du taux de chômage au taux d'emploi dans la stratégie européenne pour l'emploi ", in Zimmermann B. (éd.), Les sciences sociales à l'épreuve de l'action. Le savant, le politique et l'Europe, Éditions de la Maison des Sciences de l'Homme, Paris, p. 287-331.

Salais R., Baverez N. et Reynaud B. (1986), Linvention du chômage, PUF, Paris.

Segrestin D., (1985), Le phénomène corporatiste, Fayard, Paris.

Strauss A. (1992), La trame de la négociation. Sociologie qualitative et interactionnisme, Textes réunis et présentés par Isabelle Baszanger, L'Harmattan, Paris.

Teubner G. (1996), Droit et réflexivité, LGDJ, Paris.

Vandenbroucke F. (1999), « L'État social actif: une ambition européenne », Exposé Den Uyl, 13 décembre 1999, Amsterdam.

White H.C. (2011), Identité et contrôle. Une théorie de l'émergence des formations sociales, Éditions de l'École des Hautes Études en Sciences Sociales, Paris.

Article reçu en janvier 2014. Révision acceptée en avril 2014. 